\title{
Inflammatory Cytokines and BDNF Response to High-Intensity Intermittent Exercise: Effect the Exercise Volume
}

\author{
Carolina Cabral-Santos ${ }^{1}$, Carlos I. M. Castrillón ${ }^{2}$, Rodolfo A. T. Miranda ${ }^{2}$, \\ Paula A. Monteiro ${ }^{1}$, Daniela S. Inoue ${ }^{1}$, Eduardo Z. Campos ${ }^{1,3}$, Peter Hofmann ${ }^{4}$ and \\ Fábio S. Lira ${ }^{1 *}$ \\ ${ }^{1}$ Exercise and Immunometabolism Research Group, Departamento de Educação Física, Universidade Estadual \\ Paulista - Presidente Prudente, São Paulo, Brasil, ${ }^{2}$ Laboratório de Fisioterapia Desportiva, Faculdade de Ciências e \\ Tecnologia, Departamento de Fisioterapia, Universidade Estadual Paulista - Presidente Prudente, São Paulo, Brasil, \\ ${ }^{3}$ Department of Physical Education, Federal University of Pernambuco, Recife, Brazil, ${ }^{4}$ Exercise Physiology, Training and \\ Training Therapy Research Group, Institute of Sport Science, University of Graz, Graz, Austria
}

OPEN ACCESS

Edited by:

Gary Iwamoto,

University of Illinois at Urbana-Champaign, USA

Reviewed by:

Thomas Lowder

University of Central Arkansas, USA

James Navalta,

University of Nevada, Las Vegas, USA

*Correspondence:

Fábio S. Lira

fabiolira@fct.unesp.br

Specialty section:

This article was submitted to

Exercise Physiology,

a section of the journal

Frontiers in Physiology

Received: 16 July 2016 Accepted: 17 October 2016 Published: 04 November 2016

Citation:

Cabral-Santos C, Castrillón CIM, Miranda RAT, Monteiro PA, Inoue DS, Campos EZ, Hofmann P and Lira FS (2016) Inflammatory Cytokines and BDNF Response to High-Intensity

Intermittent Exercise: Effect the Exercise Volume.

Front. Physiol. 7:509.

doi: 10.3389/fphys.2016.00509
The purpose of this study was to compare the effects of two similar high-intensity intermittent exercises (HIIE) but different volume $1.25 \mathrm{~km}$ (HIIE1.25) and $2.5 \mathrm{~km}$ (HIIE2.5) on inflammatory and BDNF responses. Ten physically active male subjects (age $25.22 \pm 1.74$ years, body mass $78.98 \pm 7.31 \mathrm{~kg}$, height $1.78 \pm 0.06 \mathrm{~m}$, $\mathrm{VO}_{\text {2peak }} 59.94 \pm 9.38 \mathrm{ml} \cdot \mathrm{kg} \cdot \mathrm{min}^{-1}$ ) performed an incremental treadmill exercise test and randomly completed two sessions of HIIE on a treadmill (1:1 min at $\mathrm{VVO}_{2 \max }$ with passive recovery). Blood samples were collected at rest, immediately and 60-min after the exercise sessions. Serum was analyzed for glucose, lactate, IL-6, IL-10, and BDNF levels. Blood lactate concentrations was higher immediately post-exercise compared to rest (HIIE1.25: $1.69 \pm 0.26-7.78 \pm 2.09 \mathrm{mmol} \cdot \mathrm{L}^{-1}$, and HIIE2.5: $1.89 \pm 0.26-7.38 \pm 2.57$ $\left.\mathrm{mmol} \cdot \mathrm{L}^{-1}, p<0.0001\right)$. Glucose concentrations did not present changes under the different conditions, however, levels were higher 60-min post-exercise than at rest only in the HIIE1.25 condition (rest: $76.80 \pm 11.14-97.84 \pm 24.87 \mathrm{mg} \cdot \mathrm{dL}^{-1}$, $p<0.05$ ). BDNF level increased immediately after exercise in both protocols (HIIE1.25: $9.71 \pm 306-17.86 \pm 8.59 \mathrm{ng} \cdot \mathrm{mL}^{-1}$, and HIIE2.5: $11.83 \pm 5.82-22.84 \pm 10.30$ ng. $\left.\mathrm{mL}^{-1}\right)$. Although both exercises increased IL-6, level percent between rest and immediately after exercise was higher in the HIIE2.5 than HIIE1.25 (30 and 10\%; $p=0.014$, respectively). Moreover, IL-10 levels percent increase between immediately and 60-min post-exercise was higher in HIIE2.5 than HIIE1.25 (37 and 10\%; $p=0.012$, respectively). In conclusion, both HIIE protocols with the same intensity were effective to increase BDNF and IL-6 levels immediately after exercise while only IL-10 response was related to the durantion of exercise indicanting the importance of this exercise prescription variable.

Keywords: high-intensity intermittent exercise, inflammatory response, metabolic response, cytokines, low-grade inflammation 


\section{INTRODUCTION}

Classically, endurance training programs in non-athletes are aiming to promote health and prevent life style related diseases (Pedersen and Saltin, 2015). Such training programs need much time, but this does not necessarily mean that spending hours performing exercise will intensify the gains, when in fact the opposite may happen. As known for resistance exercise working until clear fatigue gives higher adaptation compared to sub-maximal number of repetitions. Similarly, Lyakh et al. (2015) discusses that a significant decrease in total training volume allows to focus on qualitative aspects of a training process.

A significantly reduced training volume increases the ability to recover more quickly, although the stimulus for the muscles may be even highly intense. HIIE is characterized by repeated bouts of short-duration (30-60 s) exercises performed at high to severe intensity (i.e., $\geq 90 \%$ of $\mathrm{VO}_{2 \max }$ ) interspersed by short periods of passive or active recovery (Zwetsloot et al., 2014). This method is considered a time-efficient way to improve health outcomes. Furthermore, Cockcroft et al. (2015) showed that a single bout of HIIE $(8 \times 1$-min at $90 \%$ of the peak power, interspersed by $1.25 \mathrm{~min}$ recovery at $20 \mathrm{~W}$ ) is an effective alternative to moderate intensity exercise to improve glucose tolerance, insulin sensitivity, and fat oxidation immediately after exercise. Studies have shown that high intensity intermittent exercise (HIIE) alone or combined with strength exercise promoted changes in metabolic and inflammatory responses (Meckel et al., 2009, 2011; Zwetsloot et al., 2014; Cabral-Santos et al., 2015; Lira et al., 2015; Wadley et al., 2016). To induce specific acute physiological responses an optimal ratio of intensity and duration of high intensity intervals and recovery as well as the resulting mean workload have to be optimized aiming for specific training adaptations (Tschakert and Hofmann, 2013).

Brain derived neurotrophic factor (BDNF), a neurotrophin family member who is involved in neuroprotection and neurogenesis, has several actions on cell functions, including energy metabolism, promoting glucose uptake via mitochondrial biogenesis, contributing to cellular homeostasis, particularly in the central nervous system (CNS; Marosi and Mattson, 2014). Saucedo Marquez et al. (2015) demonstrated that only one HIIE session was able to increase BDNF levels immediately after exercise performing a protocol with intervals of $1 \mathrm{~min}$ at $90 \%$ of maximal work load, altering with 1 min active rest (low load) during 20 min but a duration effect was not investigated.

Additionally, Monocyte Chemo-attractant Protein-1 (MCP-1) is related to inflammatory mediators and is a necessary component of the inflammatory response required for adipose tissue protection (Cranford et al., 2015). Combining exercise and dietary intake restriction is likely to prevent an influx of macrophages by reducing the number of fat cells (Ko and Kim, 2013; Ahn and Kim, 2014). Maharaj et al. (2016) observed a significant elevation across time in plasma MCP-1 and IL-6 after aerobic exercise (30-min bout performed at $75 \% \mathrm{VO}_{2 \max }$ ), suggesting that exercise could mediate an innate immune response related to monocyte recruitment and inflammatory mediators.
Besides health benefits, acute exercise enhances interleukin 6 (IL-6) concentration by increasing its production in skeletal muscle, that acts in the regulation of muscle energetic status (Pedersen and Febbraio, 2009). Additionally, IL-6 promotes enhancements in anti-inflammatory cytokines, such as interleukin 10 (IL-10), related to prevent the exacerbation of the pro-inflammatory milieu, blocking a possible persistent inflammatory status, and reduces tumor necrosis factor alpha (TNF- $\alpha$; Pedersen and Febbraio, 2009; Rosa Neto et al., 2011; Lira et al., 2012). Recently we compared the inflammatory response of $\operatorname{HIIE}\left(1: 1\right.$ at $100 \%$ at $\left.\mathrm{sVO}_{2 \text { peak }}\right)$ and moderate-intensity continuous exercise $\left(70 \%\right.$ of $\left.\mathrm{sVO}_{2 \text { peak }}\right)$ with matched volume $(5 \mathrm{~km})$, and showed that both exercise protocols promoted an anti-inflammatory response, by augmenting IL-10 and the IL10/TNF- $\alpha$ ratio (Cabral-Santos et al., 2015). This effects can be utilized as strategies for different populations, such as in patients suffering from obesity, diabetes, or dyslipidemia. From a practical point of view it is interesting to establish the optimal duration of such a HIIE to be sufficient to augment an anti-inflammatory response. Thus, the present study aimed to determine whether the same exercise intensity and mode (HIIE) but different duration and degree of fatigue (1.25- and $2.5-\mathrm{km})$ may induce similar anti-inflammatory (IL-6, IL-10, MCP-1) and metabolic (lactate and glycemic) responses in young men.

\section{MATERIALS AND METHODS}

\section{Participants}

Ten physically active male subjects volunteered for the present study. They presented a health and neuromuscular status that ensured their ability to complete the study protocol. All procedures performed in the study were approved by the Research Ethics Committee for studies involving human participants of the Universidade Estadual Paulista (UNESP) campus Presidente Prudente/SP, and were in accordance with the ethical standards of World Medical Association Declaration of Helsinki. Written informed consent was obtained from all subjects after they had been informed about the purpose and risks of the study.

Before conducting the study we checked the sample size needed ( $n=10$ ) using the $\mathrm{G}^{*}$ Power 3.1 software (Düsseldorf, Germany) to guarantee an $80 \%$ power and a $5 \%$ significance level based on a study that measured the IL- 6 pre and immediately post exercise using a similar protocol from a previous study (Cabral-Santos et al., 2015).

\section{Bioelectrical Impedance}

Bioelectrical Impendence in individuals was measured using the octopolar InBody 720 Composition Analyzer (Copyright ${ }^{\circledR}$, 1996-2006, by Biospace Corporation, USA). The participant's age, sex, and height were entered into the machine. The participants stood barefoot on the metal footplate and held the handles with their arms relaxed by their sides. Once impedance was measured, the results of fat mass and \%body fat was printed. All anthropometric measurements were checked by the same person throughout the study to minimize interpersonal variations. Participants were asked to abstain from eating or 
drinking for at least $2 \mathrm{~h}$ as well as to refrain from moderate or vigorous exercise for $24 \mathrm{~h}$ before all testing. They were told to obtain a restful night's sleep, remain well-hydrated, refrain from alcohol, and eat a regular meal in the morning before testing, all according to the manufacturer's recommendations.

\section{Maximal Endurance Running Test}

The subjects performed an incremental test to volitional fatigue (Panissa et al., 2013). The initial treadmill (Inbramed, modelo MASTER CI, Brazil) speed was set at $8.0 \mathrm{~km} \cdot \mathrm{h}-1^{-1}$ with $1 \%$ inclination, and it was increased by $1 \mathrm{~km} \cdot \mathrm{h}-1^{-1}$ after each 2 -min until the participant could no longer continue. Strong verbal encouragement was given during the test. The oxygen uptake was measured (Quark PFT, Cosmed, Rome, Italy) throughout the test and the average of the last $30 \mathrm{~s}$ was defined as peak oxygen uptake $\left(\mathrm{VO}_{2 \text { peak }}\right)$. The speed associated with $\mathrm{VO}_{2 \text { peak }}$ $\left(\mathrm{sVO}_{2 \text { peak }}\right)$ was assumed as the speed at the last stage completed or when the subject was not able to finish the 2-min stage, the speed was expressed according to the permanence time in the last stage, determined as the following: $\mathrm{sVO}_{2 \text { peak }}=$ speed of last stage complete $+[($ time (seconds) performed in the last stage/120)* 1$]$ (Kuipers et al., 1985). Heart rate was also continuously recorded throughout the tests (Polar Vantage NV, Electro Oy, Finland). The 6-20 Borg scale (Borg, 1982) was used to obtain the rating of perceived exertion during the test.

\section{High-Intensity Intermittent Exercise}

After the incremental test, the subjects completed two experimental protocols, applied in randomized cross-over order sessions, separated by at least $72 \mathrm{~h}$. The protocol was performed intermittently with subjects running on a treadmill for $1 \mathrm{~min}$ at $100 \%$ at $\mathrm{sVO}_{2 \text { peak }}$, interspersed by $1 \mathrm{~min}$ of passive recovery (which subjects jumped to the side of the treadmill by holding onto the bars for the recovery minute and remained standing or seated). They ran until they had completed the 1.25 or $2.5 \mathrm{~km}$ distance. For both exercise trials, the subjects performed a warm-up consisting of running at $50 \%$ of $\mathrm{sVO}_{2 \text { peak }}$ for $5 \mathrm{~min}$, at $1 \%$ inclination, and after that thefirst- 1-min interval exercise bout was started.

Due to the influence of time of day, all tests took place at the same time of the day (between 10:00 a.m. and 12:00 p.m.), at an average temperature between 20 and $24^{\circ} \mathrm{C}$. The subjects were instructed to abstain from strenuous exercise for at least $24 \mathrm{~h}$ prior to each exercise session, and were encouraged to maintain their usual nutritional and hydration routines. Moreover, they were also request not to ingest stimulants (tea, coffee, soda, chocolate, chocolate powder), or alcoholic beverages during this period.

\section{Dietary Intake Assessment}

Diet was not standardized. However, participants were required to eat $3 \mathrm{~h}$ prior to all testing sessions. Participants were instructed by a nutritionist how to complete the food records and were required to record all foods consumed in last night and on the day of each testing session. Nutrition data was analyzed for energy intake and macronutrient distribution using the
NutWin software version 1.5 (Programa de Apoio à Nutrição, Universidade Federal de São Paulo, Brazil, 2002).

\section{Blood Sampling and Analyses}

The blood samples were collected by a trained nurse at rest, immediately and $60 \mathrm{~min}$ after acute exercise sessions during HIIE1.25 and HIIE2.5. The blood samples $(15 \mathrm{ml})$ were immediately allocated into two $5 \mathrm{~mL}$ vacutainer tubes (Becton Dickinson, BD, Juiz de Fora, MG, Brazil) containing EDTA for plasma separation and one $5 \mathrm{~mL}$ dry vacutainer tube for serum separation. The tubes were centrifuged at $3.500 \mathrm{rpm}$ for $15 \mathrm{~min}$ at $4^{\circ} \mathrm{C}$, and plasma and serum samples were stored at $-20^{\circ} \mathrm{C}$ until analysis. Cytokines IL-6, IL-10, BDNF, and MCP-1 were assessed using ELISA commercial kits (R\&D Systems, 614 McKinley Place NE, Minneapolis, MN 55413, USA). Glucose and lactate were assessed using commercial kits (Labtest ${ }^{\circledR}$, São Paulo, Brazil).

\section{Statistical Analysis}

Data normality were verified using the Shapiro-Wilk test and descriptive data are shown as means and standard deviation. The independent $t$-test was used to compare the differences in metabolic variables at baseline between the two protocols.

One-way analysis of variance (ANOVA), with Tukey's post-hoc analysis were used to examine differences in metabolic variables at different moments (at rest, immediately after and $60 \mathrm{~min}$ after exercise) of each HIIE1.25 and HIIE2.5 effort. The same analysis was used to compare the differences $(\Delta)$ in metabolic variables between the HIIE1.25 and HIIE2.5. The repeated measures analysis was used to compare the interaction 2 (HIIT: $1.25,2.5$ ) $\times 3$ (time: pre, post, 60 post). Statistical significance was set at $5 \%$ for all the analyses and the calculations were conducted using SPSS, version 17.0 (SPSS Inc., Chicago, IL).

\section{RESULTS}

Table 1 presents the subjects characteristics and mean values of both protocols of this study.

The total food intake from last dinner and breakfast (at least $3 \mathrm{~h}$ before the test), expressed in $\mathrm{kcal}$, were not significantly different between both conditions (HIIE1.25: $980 \pm 288 \mathrm{kcal}$ vs. HIIE2.5: $1048 \pm 198 \mathrm{kcal} ; p=0.731)$ and the macronutrient distribution such as carbohydrates (HIIE1.25 $=124 \pm 54$ vs. HIIE2.5 $=101 \pm 51$ grams, $p=0.417$ ), protein (HIIE1.25 = $54 \pm 35$ vs. HIIE2.5 $=60 \pm 22$ grams, $p=0.813$ ), and lipids (HIIE1.25 $=29 \pm 13$ vs. HIIE2.5 $=41 \pm 16$ grams, $p=0.190$ ).

The concentration of glucose, lactate, IL-6, IL-10, BDNF, and MCP-1 at rest, immediately, and $60 \mathrm{~min}$ after the exercise, are presented in Table 2 and Figure 1. The variables analyzed were similar at baseline. Both HIIE exercises increased lactate and IL-6 concentration immediately after the exercise, while only the HIIE2.5 elevated IL-10 ( $p=0.023)$. Compared with at rest, glucose remained elevated $60 \mathrm{~min}$ after the HIIE1.25 ( $p=0.007)$, and IL- 6 after the HIIE2.5 $(p=0.019)$.

Table 3 shows the differences between the HIIE1.25 and HIIE2.5 for glucose, IL-10, IL-6, lactate, BDNF, and MCP-1, as well as, differences between time points (rest, immediately and $60 \mathrm{~min}$ ) for glucose, IL-10, IL-6, lactate, BDNF, and MCP-1 too. 
TABLE 1 | Subjects characteristics $(n=10)$.

\begin{tabular}{|c|c|}
\hline Variable & Mean $\pm S D$ \\
\hline Age (years) & $25.22 \pm 1.74$ \\
\hline Body Mass (kg) & $78.98 \pm 7.31$ \\
\hline Height (m) & $1.78 \pm 0.06$ \\
\hline $\mathrm{BMI}\left(\mathrm{kg} \cdot \mathrm{m}^{2}\right)$ & $24.85 \pm 2.00$ \\
\hline Fat Mass (\%) & $17.93 \pm 4.69$ \\
\hline $\mathrm{VO}_{2}$ peak $\left(\mathrm{ml} \cdot \mathrm{kg} \cdot \mathrm{min}^{-1}\right)$ & $59.94 \pm 9.38$ \\
\hline $\mathrm{sVO}_{2}$ peak $\left(\mathrm{km} \cdot \mathrm{h}^{-1}\right)$ & $13.61 \pm 1.06$ \\
\hline Duration session $\mathrm{HIIE}_{1.25}$ (min) & $10.08 \pm 0.81$ \\
\hline Duration session $\mathrm{HIIE}_{2.5}(\mathrm{~min})$ & $21.15 \pm 1.62$ \\
\hline Bouts per session $\mathrm{HIIE}_{1.25}$ & $5.54 \pm 0.40$ \\
\hline Bouts per session $\mathrm{HIIE}_{2.5}$ & $11.08 \pm 0.81$ \\
\hline Heart rate rest (bpm) & $65.40 \pm 7.26$ \\
\hline Heart rate final $\mathrm{HIIE}_{1.25}(\mathrm{bpm})$ & $170.0 \pm 6.94$ \\
\hline Heart rate final $\mathrm{HIIE}_{2.5}(\mathrm{bpm})$ & $175.7 \pm 9.09$ \\
\hline
\end{tabular}

$\mathrm{BMI}$, Body Mass Index; $\mathrm{VO}_{2}$ max, maximal oxygen uptake; $\mathrm{sVO}_{2 \max }$, speed associated with maximal oxygen uptake; bpm, beat per minutes.

TABLE 2 | Alterations of metabolic variables after HIIE 1.25 and $2.5 \mathrm{~km}$ $(n=10)$.

\begin{tabular}{|c|c|c|c|}
\hline $1.25 \mathrm{~km}$ & $\begin{array}{c}\text { Rest } \\
\text { mean }(S D)\end{array}$ & $\begin{array}{c}\text { Immediately } \\
\text { mean (SD) }\end{array}$ & $\begin{array}{c}60 \min \\
\text { mean (SD) }\end{array}$ \\
\hline Glucose (mg/dL) & $76.80(11.14)$ & $72.77(17.32)$ & $97.84(24.87)^{\mathrm{a}, \mathrm{b}}$ \\
\hline Lactate (mmol/L) & $1.69(0.26)$ & $7.78(2.09)^{\mathrm{a}}$ & $1.79(0.42)^{\mathrm{b}}$ \\
\hline IL-6 (pg/mL) & $1.51(0.34)$ & $1.67(0.31)^{\mathrm{a}}$ & $1.72(0.34)$ \\
\hline IL-10 (pg/mL) & $1.51(0.86)$ & $1.67(0.56)$ & $2.09(0.80)$ \\
\hline MCP-1 (pg/mL) & $4.81(0.72)$ & $5.46(1.76)^{\mathrm{a}}$ & $4.74(0.72)^{\mathrm{b}}$ \\
\hline BDNF (ng/mL) & $9.71(3.06)$ & $17.86(8.59)^{\mathrm{a}}$ & $8.82(2.74)^{\mathrm{b}}$ \\
\hline $2.5 \mathrm{~km}$ & $\begin{array}{c}\text { Rest } \\
\text { mean }(S D)\end{array}$ & $\begin{array}{c}\text { Immediately } \\
\text { mean }(S D)\end{array}$ & $\begin{array}{c}60 \min \\
\text { mean (SD) }\end{array}$ \\
\hline Glucose (mg/dL) & $86.92(12.77)$ & 81.39 (12.67) & 87.45 (11.29) \\
\hline Lactate (mmol/L) & $1.89(0.26)$ & $7.38(2.57)^{\mathrm{a}}$ & $1.97(0.49)^{b}$ \\
\hline IL-6 (pg/mL) & $1.55(0.42)$ & $2.03(0.59)^{a}$ & 1.79 (0.62) \\
\hline IL-10 (pg/mL) & $2.16(0.43)$ & $2.96(1.04)^{\mathrm{a}}$ & $2.04(1.04)^{b}$ \\
\hline MCP-1 (pg/mL) & $5.14(1.01)$ & $5.05(0.87)$ & $4.95(0.75)$ \\
\hline BDNF (ng/mL) & $11.83(5.82)$ & $22.84(10.30)^{a}$ & $8.40(3.04)^{b}$ \\
\hline
\end{tabular}

${ }^{a}$ Tukey's post-hoc test with $p<0.05$ compared to rest; ${ }^{b}$ Tukey's post-hoc test with $p<0.05$ compared between Immediately with $60 \mathrm{~min}$.

The variables IL-6 and IL-10, showed interaction between the group (HIIE1.25 vs. 2.5) and the time (rest, immediately and $60 \mathrm{~min})$.

\section{DISCUSSION}

Recently, a study by Wadley et al. (2016) suggest that the magnitude of increase in cytokines in response to exercise is dependent on exercise intensity. In our study we questioned whether there is a threshold like level of HIIE intensity vs. time combination required to observe such effect. The main finding of the present study suggests that the increase in plasma concentrations of IL-10 occurs in a duration-dependent manner, as that same intensity in the task but twice the duration gave differences in the response profile. HIIE2.5 maintained temporal differences that induced higher IL-10 levels (indicated by the highest peak in the phase immediately following exercise) when compared to HIIE1.25, which only presented a trend for an increase. However, after 60-min, the IL-10 levels had returned to baseline values in both protocols.

High-intensity intermittent training has been shown to be effective for improving body composition parameters, physical fitness, and lipid profile (Martins et al., 2016). Acute studies have proposed that HIIE increases glucose tolerance and insulin sensitivity (Cockcroft et al., 2015). This response to HIIE is due to a closed integration between the different body systems, especially the metabolic and immune systems. Recently, BDNF, more known for its neuro-regenerative function, has also been related to metabolism, with energy challenges leading to increased concentrations (Marosi and Mattson, 2014). In the present study, both protocols led to energy stress, demonstrated by lactate which increased in both HIIE1.25 and HIIE2.5 immediately after exercise if standardized to basal values justifying, at least, the BDNF behavior immediately and $60 \mathrm{~min}$ after exercise (Table 2). These results are according to studies by Griffin et al. (2011) and Saucedo Marquez et al. (2015). However, there was no difference between HIIE1.25 and HIIE2.5 in BDNF response, even though the duration of HIIE was set differently in both protocols. Ferris et al. (2007) demonstrated that BDNF increase was higher with higher exercise intensity, independent of the duration of exercise execution (HIIE1.25: $10.8 \pm 0.81 \mathrm{~min}$ vs. HIIE2.5: $21.15 \pm 1.62 \mathrm{~min}$ ). In fact, HIIE has a higher "stressing nature" than continuous aerobic exercise because of its anaerobic components which are suggested to lead to cerebral hypoxia that can be a trigger to augment BDNF release by the brain itself (Rasmussen et al., 2009) as BDNF is related to cell survival and neuro-protection. On the other hand, BDNF levels decreased after $60 \mathrm{~min}$, possibly because BDNF was taken up by muscle tissue to support lipid metabolism (Rasmussen et al., 2009).

Regarding metabolic response, lactate has been proposed as an important glycolytically produced metabolite that is most likely released because of increased or accelerated anaerobic glycolysis and stress response (Garcia-Alvarez et al., 2014). All participants demonstrated an increase in lactate concentration during the exercise session in both conditions. When the rate of glucose metabolism exceeds the oxidative capacity of the mitochondria (Dienel, 2013), lactate assists as a critical buffer, allowing glycolysis to rapidly produce significant amounts of energy. The higher variation in glucose after the HIIE1.25 compared to the HIIE2.5 may indicate that shorter HIIE stimulated glyconeogenesis (through an increase in catecholamines); however, as the exercise was very short, this glucose was probably not used during the exercise (Lira et al., 2015). On the other hand, the clearance of lactate during the recovery period was not measured, limiting a comparison between lactate levels after both HIIEs.

Recently, we verified that $5 \mathrm{~km}$ HIIE augments the anti-inflammatory status (Cabral-Santos et al., 2015). However, 


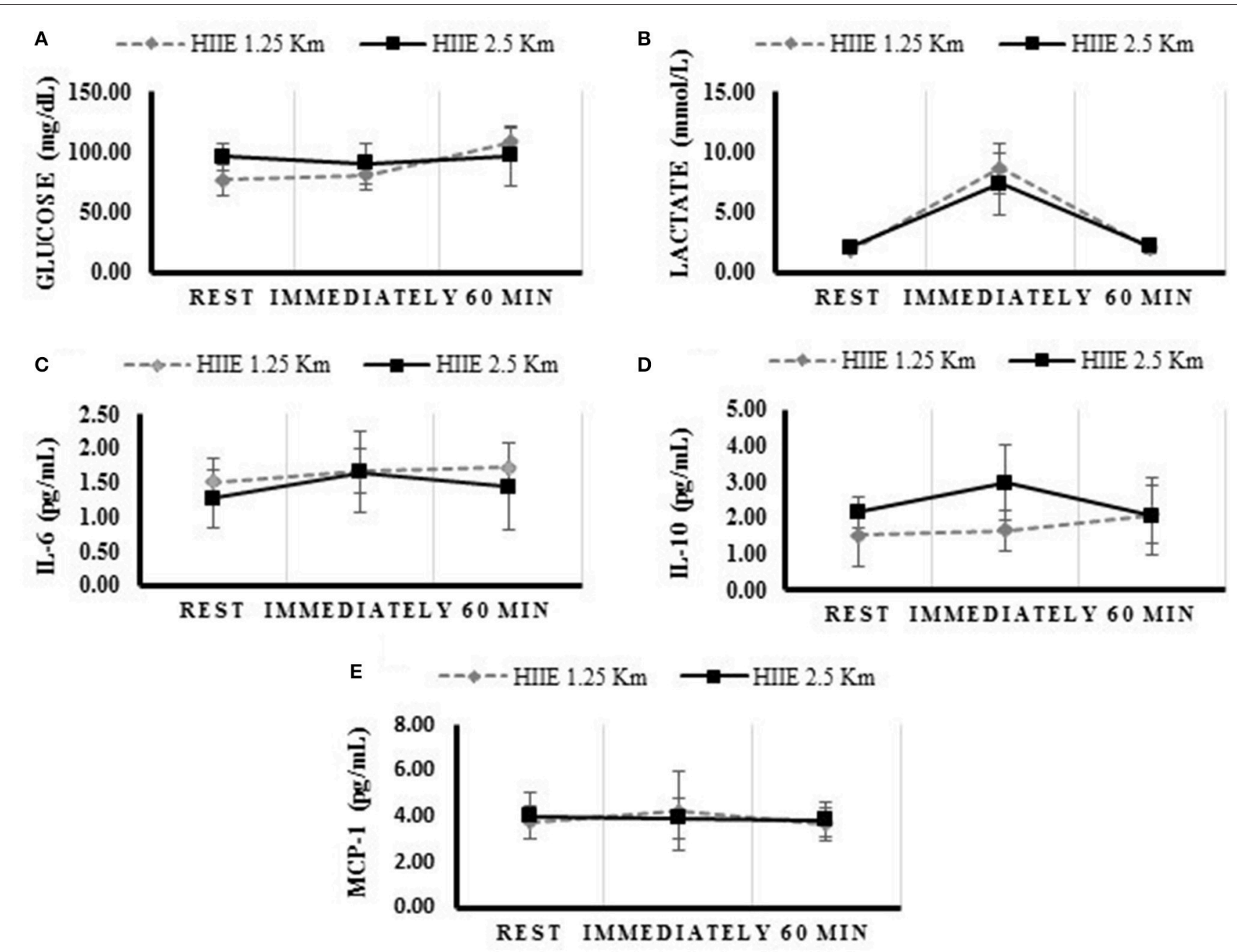

FIGURE 1 | Concentrations of metabolic and inflammatory profile during rest, immediately and 60 min after the HIIE 1.25 and HIIE $2.5 \mathrm{~km}$. Glucose (A), Lactate (B), Interleukine 6 (C), Interleukine 10 (D), Monocyte Chemo-attractant Protein 1 (E).

no study has verified whether even lower HIIE duration elicits the same anti-inflammatory response. This result could be important for training prescription, especially since the rationale for HIIE is its time-efficiency.

Studies generally adopt different HIIE protocols to evaluate anti-inflammatory effects. Lira et al. (2015) submitted athletes to 4 sessions of the Wingate test at $100 \%$ of $\mathrm{VO}_{2 \text { peak }}$ and observed an increase in IL-10 levels, inducing beneficial alterations in the resting inflammatory profile. Dorneles et al. (2016) demonstrated that $10 \times 60 \mathrm{~s}$ at $85-90 \%$ of maximal aerobic power, separated by 75 -s at $50 \%$ of maximal aerobic power (which gives a mean workload of $65.6-67.8 \%$ of $\mathrm{P}_{\max }$ ), was able to induce a progressive elevation of IL- 6 and IL-10 levels immediately and 30-min post exercise in both lean and overweight-obese subjects. These results suggest an important implication of this exercise in the control of chronic low-grade inflammation in obesity. Furthermore, Wadley et al. (2016) compared untrained males who undertook three exercise bouts: HIIE $\left(10 \times 1-\min\right.$ at $\left.90 \% \mathrm{VO}_{2 \max }\right)$ and two energy-matched steady-state cycling bouts at a moderate $(60 \%$
$\left.\mathrm{VO}_{2 \max }, 27 \mathrm{~min}\right)$ and high $\left(80 \% \mathrm{VO}_{2 \max }, 20 \mathrm{~min}\right)$ intensity, and showed that IL-6 and IL-10 increased after $30 \mathrm{~min}$ in both HIIE and high intensity steady-state cycling, demonstrating similar inflammatory and oxidative stress responses. Taken together, these studies showed that the acute anti-inflammatory response in HIIE seems to be intensity-dependent (CabralSantos et al., 2015; Dorneles et al., 2016). However, our results contradict, at the least, this perspective showing that also the duration of an exercise bout may be an important variable.

As can be seen in Table 3, the delta (measure of difference in final concentrations compared with the basal; $\Delta$ ) in IL-6 changes from rest to immediately after exercise was higher for HIIE2.5 than HIIE1.25, demonstrating that IL-6 level increases may be dependent on exercise duation. Additionally, IL-10 was shown to be related to the volume of HIIE, as this variable tended to be higher in HIIE2.5 than HIIE1.25 $(p<0.059)$ between rest and immediately after exercise, although at immediately and 60 min post exercise a negative delta to HIIE2.5 and a positive delta 
TABLE 3 | Difference between the deltas of the metabolic variables of HIIE 1.25 and $2.5 \mathrm{~km}(n=10)$.

\begin{tabular}{|c|c|c|c|c|c|c|c|}
\hline & & $\begin{array}{l}\text { HIIE } 1.25 \text { km } \\
\Delta \text { mean }(S D)\end{array}$ & $\begin{array}{l}\text { HIIE } 2.5 \mathrm{~km} \\
\Delta \text { mean }(S D)\end{array}$ & Effect & $\boldsymbol{F}$ & $\boldsymbol{P}$ & Effect size \\
\hline \multirow[t]{3}{*}{ Glucose (mg/dL) } & Rest and immediately & $-3.27(13.62)$ & $-5.54(18.30)$ & Group & 8.450 & 0.003 & 0.514 \\
\hline & Rest and $60 \mathrm{~min}$ & $21.78(18.13)$ & $0.54(10.42)$ & Time & 8.241 & 0.011 & 0.326 \\
\hline & Immediately and 60 min & 25.05 (22.19) & $6.07(11.30)$ & Group $\times$ Time & 1.444 & 0.246 & 0.078 \\
\hline \multirow[t]{3}{*}{ Lactate (mmol/L) } & Rest and Immediately & $6.09(2.05)$ & $4.75(3.38)$ & Group & 45.396 & $<0.001$ & 0.842 \\
\hline & Rest and 60 min & $0.11(0.50)$ & $0.08(0.43)$ & Time & 80.498 & $<0.001$ & 0.817 \\
\hline & Immediately and $60 \mathrm{~min}$ & $-5.98(1.77)$ & $-4.67(3.17)$ & Group $\times$ Time & 1.234 & 0.281 & 0.064 \\
\hline \multirow[t]{3}{*}{ IL-6 (pg/mL) } & Rest and Immediately & $0.15(0.14)$ & $0.47(0.31)$ & Group & 14.495 & $<0.001$ & 0.659 \\
\hline & Rest and 60 min & $0.21(0.39)$ & $0.25(0.25)$ & Time & 12.384 & 0.003 & 0.436 \\
\hline & Immediately and 60 min & $0.05(0.35)$ & $-0.23(0.32)$ & Group x Time & 6.764 & 0.019 & 0.297 \\
\hline \multirow[t]{3}{*}{ IL-10 (pg/mL) } & Rest and Immediately & $0.15(0.42)$ & $0.80(0.85)$ & Group & 4.226 & 0.035 & 0.360 \\
\hline & Rest and 60 min & $0.58(0.96)$ & $-0.11(0.98)$ & Time & 4.649 & 0.047 & 0.225 \\
\hline & Immediately and 60 min & $0.42(0.78)$ & $-0.91(1.19)$ & Group x Time & 8.904 & 0.009 & 0.358 \\
\hline \multirow[t]{3}{*}{ MCP-1 (pg/mL) } & Rest and Immediately & $0.65(1.69)$ & $-0.10(0.41)$ & Group & 1.559 & 0.253 & 0.221 \\
\hline & Rest and 60 min & $-0.06(0.16)$ & $-0.19(0.43)$ & Time & 1.104 & 0.314 & 0.084 \\
\hline & Immediately and 60 min & $-0.71(1.70)$ & $-0.09(0.23)$ & Group $\times$ Time & 1.113 & 0.312 & 0.085 \\
\hline \multirow[t]{3}{*}{$\mathrm{BDNF}(\mathrm{ng} / \mathrm{mL})$} & Rest and Immediately & $9.02(7.05)$ & $11.01(4.99)$ & Group & 24.634 & $<0.001$ & 0.755 \\
\hline & Rest and $60 \mathrm{~min}$ & $-1.08(2.60)$ & $-4.27(6.01)$ & Time & 45.097 & $<0.001$ & 0.726 \\
\hline & Immediately and 60 min & $-10.11(8.32)$ & $-15.2(9.75)$ & Group $\times$ Time & 1.116 & 0.305 & 0.062 \\
\hline
\end{tabular}

Bold values $=$ significant difference with $p<0.05$.

to HIIE1.25 was found showing that IL-10 was still increasing $(p<0.012)$.

IL-6 is an important mediator involved in the regulation of the acute-phase response to injury and infection (Heinrich et al., 2003). In muscle cells, contraction leads to the activation of the mitogen-activated protein kinase (MAPK) and Janus kinase (JAK)-signal transducers and activators of transcription (STAT) cascade phosphorylation (Wunderlich et al., 2013), ending with IL-6 transcription in skeletal muscle. It is important to note that the IL- 6 produced and secreted by skeletal muscle has beneficial effects on metabolism, since this response exerts effects on GLUT4 translocation in the muscle that increases glycogen synthesis, insulin sensitivity in central, and peripheral organs and enhances lipid oxidation in the skeletal muscle (Al-Khalili et al., 2006; Pal et al., 2014; Cron et al., 2016), in order to provide fuel to supply the skeletal muscle during activity.

Nieman et al. (2015) showed that prolonged and intense running on a treadmill at $70 \% \mathrm{VO}_{2 \max }$ until exhaustion increased muscle mRNA expression, muscle protein content, and plasma levels for IL-6, IL-8, and MCP-1, and in addition post-run muscle glycogen concentrations were negatively correlated with changes in muscle IL- 6 protein content. Concurring with these data, the present study demonstrated a volume dependent increase in IL-6 levels.

Additionally, IL-6 modulates anti-inflammatory processes, as this cytokine is able to increase the production of antiinflammatory cytokines such as IL-10 and IL-1ra (Petersen and Pedersen, 2005). IL-10 is important in the anti-inflammatory response and promotes the preservation of $\mathrm{IkB}$, thereby causing inhibition of nuclear transcription factor kappa B (NF-kB), the main transcription factor of TNF- $\alpha$ (Petersen and Pedersen, 2005) - a cytokine with a pro-inflammatory character that acts on the regulation of insulin sensitivity and also induces lipolysis. Recently, Cabral-Santos et al. (2015) showed markedly increased IL-6 and IL-10 levels after HIIE (1:1-min at $\mathrm{vVO}_{2 \max }, 5 \mathrm{~km}$ run) in trained subjects, suggesting an important role of HIIE in blocking a possible persistent inflammatory milieu.

Our data did not show changes in the concentrations of MCP-1. Further studies are needed to better understand the mechanisms involved and the effects of different exercise modalities, intensities and volumes.

Beside the effects of different HIIE volumes on antiinflammatory status, highlighting its potential as a feasible form of exercise prescription for coaches who want to avoid overtraining or overreaching status, low volume HIIE can be performed, since no modifications in inflammatory parameters were found after the low volume HIIE in our study. Nevertheless, other studies are warranted to verify the chronic effects of low volume HIIE on other parameters to confirm its utility. However, as shown by Wallner et al. (2013) even high intensity intervals at $\mathrm{vVO}_{2 \max }$ may lead to rather low lactate responses in case the overall mean load is low and the duration of the strenuous interval bouts is short showing the need for further studies systematically varying the variables of interval exercise.

In conclusion, this study provides preliminary evidence to suggest that HIIE may be an effective intervention strategy for promoting elevations in BDNF concentrations and in the inflammatory response of IL-6, thus both protocols were rated 
equally. Furthermore, the volume of HIIE seems to influence the time-response of IL-10 levels of plasma, once in the lowest volume the response of IL-10 was sustained. Both protocols are suggested to lead to improvements in the anti-inflammatory status although the maximal/optimal number of repetitions (duration) as well as the impact of them mean exercise intensity is not clear at the moment.

\section{AUTHOR CONTRIBUTIONS}

Substantial contributions to the conception or design of the work-CCS, CIMC, and RM. Analysis and interpretation of data for the work-PM and FL. Drafting the work or revising

\section{REFERENCES}

Ahn, N., and Kim, K. (2014). Combined influence of dietary restriction and treadmill running on MCP-1 and the expression of oxidative stress-related mRNA in the adipose tissue in obese mice. J. Exerc. Nutr. Biochem. 18, 311-318. doi: $10.5717 /$ jenb.2014.18.3.311

Al-Khalili, L., Bouzakri, K., Glund, S., Lönnqvist, F., Koistinen, H. A., and Krook, A. (2006). Signaling specificity of interleukin-6 action on glucose and lipid metabolism in skeletal muscle. Mol. Endocrinol. 20, 3364-3375. doi: 10.1210/me.2005-0490

Borg, G. A. (1982). Psychophysical bases of perceived exertion. Med. Sci. Sports Exerc. 14, 377-381.

Cabral-Santos, C., Gerosa-Neto, J., Inoue, D. S., Panissa, V. L., Gobbo, L. A., Zagatto, A. M., et al. (2015). Similar anti-inflammatory acute responses from moderate-intensity continuous and high-intensity intermittent exercise. $J$. Sports Sci. Med. 14, 849-856.

Cockcroft, E. J., Williams, C. A., Tomlinson, O. W., Vlachopoulos, D., Jackman, S. R., Armstrong, N., et al. (2015). High intensity interval exercise is an effective alternative to moderate intensity exercise for improving glucose tolerance and insulin sensitivity in adolescent boys. J. Sports Sci. Med. 18, 720-724. doi: 10.1016/j.jsams.2014.10.001

Cranford, T. L., Enos, R. T., Velázquez, K. T., McClellan, J. L., Davis, J. M., Singh, U. P., et al. (2015). Role of MCP-1 on inflammatory processes and metabolic dysfunction following high-fat feedings in the FVB/N strain. Int. J. Obes. Dec 1:244. doi: 10.1038/ijo.2015.244

Cron, L., Allen, T., and Febbraio, M. A. (2016). The role of gp130 receptor cytokines in the regulation of metabolic homeostasis. J. Exp. Biol. 219(Pt 2), 259-265. doi: 10.1242/jeb.129213

Dienel, G. A. (2013). Astrocytic energetics during excitatory neurotransmission: what are contributions of glutamate oxidation and glycolysis? Neurochem. Int. 63, 244-258. doi: 10.1016/j.neuint.2013.06.015

Dorneles, G. P., Haddad, D. O., Fagundes, V. O., Vargas, B. K., Kloecker, A., Romão, P. R., et al. (2016). High intensity interval exercise decreases IL-8 and enhances the immunomodulatory cytokine interleukin-10 in lean and overweight-obese individuals. Cytokine 77, 1-9. doi: 10.1016/j.cyto.2015.10.003

Ferris, L. T., Williams, J. S., and Shen, C. L. (2007). The effect of acute exercise on serum brain-derived neurotrophic factor levels and cognitive function. Med. Sci. Sports Exerc. 39, 728-734. doi: 10.1249/mss.0b013e31802f04c7

Garcia-Alvarez, M., Marik, P., and Bellomo, R. (2014). Sepsis-associated hyperlactatemia. Crit. Care 18, 503. doi: 10.1186/s13054-014-0503-3

Griffin, E. W., Mullally, S., Foley, C., Warmington, S. A., O’mara, S. M., and Kelly A. M. (2011). Aerobic exercise improves hippocampal function and increases BDNF in the serum of young adult males. Physiol. Behav. 104, 934-941. doi: 10.1016/j.physbeh.2011.06.005

Heinrich, P. C., Behrmann, I., Haan, S., Hermanns, H. M., Müller-Newen, G., and Schaper, F. (2003). Principles of interleukin (IL)-6-type cytokine signalling and its regulation. Biochem. J. 374,1-20. doi: 10.1042/bj20030407

Ko, J., and Kim, K. (2013). Effects of exercise and diet composition on expression of MCP-1 and oxidative stress-related mRNA of adipose tissue it critically for important intellectual content-DI, PH. Final approval of the version to be published-FL. All authors agree to be accountable for the content of the work.

\section{FUNDING}

This work was supported by the Fundação de Amparo à Pesquisa do Estado de São Paulo (FAPESP, Brazil) under Grant $\mathrm{n}^{\circ}$ 2013/25310-2.

\section{ACKNOWLEDGMENTS}

FL thanks Fapesp for their support (2013/25310-2). in diet-induced obese mice. J. Exerc. Nutrition Biochem. 17, 181-188. doi: 10.5717/jenb.2013.17.4.181

Kuipers, H., Verstappen, F. T. J., Keizer, H. A., Geurten, P., and van Kranenburg, G. (1985). Variability of aerobic performance in the laboratory and its physiologic correlates. Int. J. Sports Med. 6, 197-201.

Lira, F. S., Panissa, V. L., Julio, U. F., and Franchini, E. (2015). Differences in metabolic and inflammatory responses in lower and upper body highintensity intermittent exercise. Eur. J. Appl. Physiol. 115, 1467-1474. doi: 10.1007/s00421-015-3127-7

Lira, F. S., Yamashita, A. S., Rosa, J. C., Koyama, C. H., Caperuto, E. C., Batista, M. L., et al. (2012). Exercise training decreases adipose tissue inflammation in cachectic rats. Horm. Metab. Res. 44, 91-98. doi: 10.1055/s-00311299694

Lyakh, V., Mikołajec, K., Bujas, P., and Litkowycz, R. (2015). Review of Platonov's "Sports Training Periodization. General Theory and its Practical Application" - Kiev: Olympic Literature, 2013 (part two). J. Hum. Kinet. 27, 273-278. doi: 10.1515/hukin-2015-0055

Maharaj, A., Slusher, A. L., Zourdos, M. C., Whitehurst, M., Fico, B. G., and Huang, C. J. (2016). Association of calprotectin with leukocyte chemotactic and inflammatory mediators following acute aerobic exercise. Appl. Physiol. Nutr. Metab. 41, 83-87. doi: 10.1139/apnm-2015-0385

Marosi, K., and Mattson, M. P. (2014). BDNF Mediates Adaptive Brain and body responses to energetic challenges. Trends Endocrinol. Metab. 25, 89-98. doi: 10.1016/j.tem.2013.10.006

Martins, C., Kazakova, I., Ludviksen, M., Mehus, I., Wisloff, U., Kulseng, B., et al. (2016). High-intensity interval training and isocaloric moderate-intensity continuous training result in similar improvements in body composition and fitness in obese individuals. Int. J. Sport Nutr. Exerc. Metab. 26, 197-204. doi: 10.1123/ijsnem.2015-0078

Meckel, Y., Eliakim, A., Seraev, M., Zaldivar, F., Cooper, D. M., Sagiv, M., et al. (2009). The effect of a brief sprint interval exercise on growth factors and inflammatory mediators. J. Strength Cond. Res. 23, 225-230. doi: 10.1519/JSC.0b013e3181876a9a

Meckel, Y., Nemet, D., Bar-Sela, S., Radom-Aizik, S., Cooper, D. M., Sagiv, M., et al. (2011). Hormonal and inflammatory responses to different types of sprint interval training. J. Strength Cond. Res. 25, 2161-2169. doi: 10.1519/JSC.0b013e3181dc4571

Nieman, D. C., Zwetsloot, K. A., Meaney, M. P., Lomiwes, D. D., Hurst, S. M., and Hurst, R. D. (2015). Post-exercise skeletal muscle glycogen related to plasma cytokines and muscle IL-6 protein content, but not muscle cytokine mRNA expression. Front. Nutr. 9:27. doi: 10.3389/fnut.2015. 00027

Pal, M., Febbraio, M. A., and Whitham, M. (2014). From cytokine to myokine: the emerging role of interleukin-6 in metabolic regulation. Immunol. Cell Biol. 92, 331-339. doi: 10.1038/icb.2014.16

Panissa, V. L. G., Azevedo, N. R. M., Julio, U. F., Andreato, L. V., Pinto e Silva, C. M., Hardt, F., et al. (2013). Maximum number of repetitions, total weight lifted and neuromuscular fatigue in individuals with different training backgrounds. Biol. Sport 30, 131-136. doi: 10.5604/20831862.1044458 
Pedersen, B. K., and Febbraio, M. A. (2009). Muscle as an endocrine organ: focus on muscle-derived interleukin-6. Physiol. Rev. 88, 1379-1406. doi: 10.1152/physrev.90100.2007

Pedersen, B. K., and Saltin, B. (2015). Exercise as medicine - evidence for prescribing exercise as therapy in 26 different chronic diseases. Scand. J. Med. Sci. Sports 25(Suppl. 3), 1-72. doi: 10.1111/sms.12581

Petersen, A. M., and Pedersen, B. K. (2005). The anti-inflammatory effect of exercise. J. Appl. Physiol. 98, 1154-1162. doi: 10.1152/japplphysiol.001 64.2004

Rasmussen, P., Brassard, P., Adser, H., Pedersen, M. V., Leick, L., Hart, E., et al. (2009). Evidence for a release of brain-derived neurotrophic factor from the brain during exercise. Exp. Physiol. 94 1062-1069. doi: 10.1113/expphysiol.2009.048512

Rosa Neto, J. C., Lira, F. S., de Mello, M. T., and Santos, R. V. T. (2011). Importance of exercise immunology in health promotion. Amino Acids 41, 1165-1172. doi: 10.1007/s00726-010-0786-x

Saucedo Marquez, C. M., Vanaudenaerde, B., Troosters, T., and Wenderoth, N. (2015). High-intensity interval training evokes larger serum BDNF levels compared with intense continuous exercise. J. Appl. Physiol. 119, 1363-1373. doi: 10.1152/japplphysiol.00126.2015

Tschakert, G., and Hofmann, P. (2013). High-intensity intermittent exercise: methodological and physiological aspects. Int. J. Sports Physiol. Perform. 8, 600-610. doi: 10.1123/ijspp.8.6.600

Wadley, A. J., Chen, Y. W., Lip, G. Y., Fisher, J. P., and Aldred, S. (2016). Low volume-high intensity interval exercise elicits antioxidant and anti-inflammatory effects in humans. J. Sports Sci. 34, 1-9. doi: 10.1080/02640414.2015.1035666

Wallner, D., Simi, H., Tschakert, G., and Hofmann, P. (2013). Acute physiological response to aerobic short-interval training in trained runners. Int. J. Sports Physiol. Perform. 9, 661-666. doi: 10.1123/ijspp. 2013-0385

Wunderlich, C. M., Hövelmeyer, N., and Wunderlich, F. T. (2013). Mechanisms of chronic JAK-STAT3-SOCS3 signaling in obesity. JAKSTAT 2:e23878. doi: $10.4161 /$ jkst. 23878

Zwetsloot, K. A., John, C. S., Lawrence, M. M., Battista, R. A., and Shanely, R. A. (2014). High-intensity interval training induces a modest systemic inflammatory response in active, young men. J. Inflamm. Res. 7, 9-17. doi: 10.2147/jir.s54721

Conflict of Interest Statement: The authors declare that the research was conducted in the absence of any commercial or financial relationships that could be construed as a potential conflict of interest.

Copyright (c) 2016 Cabral-Santos, Castrillón, Miranda, Monteiro, Inoue, Campos, Hofmann and Lira. This is an open-access article distributed under the terms of the Creative Commons Attribution License (CC BY). The use, distribution or reproduction in other forums is permitted, provided the original author (s) or licensor are credited and that the original publication in this journal is cited, in accordance with accepted academic practice. No use, distribution or reproduction is permitted which does not comply with these terms. 